\title{
Synergy of Educational Tools in Teaching of a New Lecture/Lab Course on Electromagnetic Fields and Waves
}

\section{Dr. Nizami Z Vagidov, University at Buffalo, The State University of New York}

Dr. Nizami Vagidov is a Research Assistant Professor at Electrical Engineering Department of University at Buffalo, The State University of New York. He obtained his Ph.D. degree in Electrical Engineering in 2001 from the Department of Electrical and Computer Engineering, Wayne State University, Detroit, MI. His dissertation was devoted to nonconventional nanoscale semiconductor devices. He is a member of scientific societies such as IEEE, American Physical Society, and Sigma Xi. He is a co-author of three patents and he co-authored a book "Quantum Mechanics for Nanostructures", Cambridge University Press, 2010. He has more than 100 technical publications in leading scientific journals.

\section{Dr. Vladimir Mitin, University at Buffalo, SUNY}

Vladimir Mitin, SUNY Distinguished Professor at the Department of Electrical Engineering at the University at Buffalo, The State University of New York. He is a fellow of IEEE, SPIE, APS, AAAS, and IoP. He was the Chair of that Department for two terms: 2003-2006 and 2006-2009. During 1993-2003 he was a Professor in the Department of Electrical and Computer Engineering at Wayne State University in Detroit, Michigan. His fields of specialization are nanoelectronic, microelectronic and optoelectronic devices and materials. Currently he is working in the areas of design and characterization of electronic and optoelectronic devices with emphasis on light absorption and emission, energy conversion and heat dissipation. Special attention in his research is now placed on simulation, design, and characterization of nanosensors, and quantum dot infrared photodetectors and solar cells. He has more than 230 publications in refereed journals, fourteen patents, four monographs and five textbooks. He obtained his Doctor of Science degree in 1987 from the Institute of Semiconductors of Ukrainian Academy of Sciences in Kiev, Ukraine.

Prof. Athos Chariton Petrou

Dr. Joseph L Zawicki Zawicki 


\title{
Synergy of Educational Tools in Teaching of a New Lecture/Lab Course on Electromagnetic Fields and Waves
}

\begin{abstract}
Electromagnetic Fields and Waves (EFW) courses for undergraduate students are usually taught in traditional lecture style. The instructors that teach EFW courses using this conventional method encounter several problems including these:

1. There is disconnect between the mathematics used to describe electromagnetic phenomena and the physics, even when one or two chapters on mathematical topics is given at the beginning of the semester. The situation is even worse if the teachers rely on students' knowledge from math courses.

2. There is disconnect between the theory discussed in the lectures and the experiments carried out in the accompanying lab. This disconnect is made more severe by two factors: (a) sometimes the lab either precedes or lags behind the lecture material and (b) the theory and the lab are taught by two separate instructors who profess different teaching philosophies and have different ideas about what is important and what is not.

3. In the present teaching system the assessment of students' understanding of the subject is infrequent. Typically it consists of a final exam with one or two midterm exams. As a result the students do not put a uniform effort in learning during the course but, under pressure from their other courses, apply themselves to the EFW course for only a short period of time just before the exams, resulting in an uneven and incomplete learning.
\end{abstract}

To overcome the problems described above we are developing a conceptually novel onesemester EFW course for engineering junior undergraduate students and establishing a new undergraduate EFW laboratory. This course is a four credit-hour lecture/lab course (three credit hours are for the lecture component and one credit hour for the lab component of the combined course). A novel style of teaching this EFW course is based on the interactive approach experiment - theory - experiment - applications. In this approach we emphasize that:

1. Each theoretical topic, described in the lecture part of the course, is covered by a lab experiment.

2. To acquire new abstract notions the educational Java applets will be heavily employed. Java applets will be used in a way that provides only active style of learning. Java applets, as used in traditional lectures, provide only a passive experience.

3. Connected with Java applets are means for numerical solutions of electromagnetic problems. Students will be able to compare numerical results with the experimental data that they obtained carrying out lab experiments as well as with the results provided by Java applets.

4. The students' progress will be assessed each week by giving them: (a) in-class quizzes and (b) out-class quizzes using the Blackboard online system. This will ensure a uniform effort by the students which is expected to result in a better understanding of the course material.

Thus, the undergraduate engineering students through experiments, educational Java applets, and software for numerical solution actively learn the theoretical principles of EFW, apply them to real-world problems, and develop a scientific approach, which improves critical reasoning and creative thinking. 


\section{Introduction}

A novel approach is undertaken for the development of an Electromagnetic Fields and Waves (EFW) course for undergraduate engineering juniors at University at Buffalo (UB). This approach has the following three major aspects: 1) a nontraditional synergistic style of teaching which includes the following components: (a) coherent combination of lecture and lab components of the course, $(b)$ use of educational Java applets, and (c) use of numerical software for solution of EFW problems; 2) since electricity and magnetism is an experimental science, the relevant abstract notions are introduced on the basis of corresponding experiments; and 3) a unified description of the entire spectrum of electromagnetic waves is used to address interdisciplinary issues. We emphasize here that EFW is treated as an experimental science and teaching of electromagnetic theory is based essentially on experiments that students will carry out in a new EFW undergraduate lab specifically established for this course. Thus, students will not only gain theoretical knowledge from the course lectures but will also acquire important hands-on experience and develop a scientific way of thinking. This change of the teaching style of a new EFW course is characterized by the following features: (i) the hands-on experience is provided to students while carrying out seven lab experiments, (ii) day-to-day monitoring of students' learning process is monitored by using Blackboard online system, and (iii) assessment of progress in learning is done through low-stake quizzes, homework problems, lab reports, and exams.

Since the understanding of electricity and magnetism requires a certain level of mathematical knowledge, the required mathematical concepts such as vector algebra and vector calculus will be introduced and discussed gradually in step with the teaching material covered in the course. Teaching experience shows that students are more likely to learn abstract mathematical notions when these notions are applied to specific problems.

The proposed style of teaching can be described best as a synergy of educational tools, i.e. "combined action" of four components: 1. Power-point presentations; 2. Lab experiments; 3. Java applets; and 4. Numerical calculations; the word synergy originates from the Greek word "synergos" which means "working together". As it is noted in Ref. $[1,2]$ such combination of educational tools allows students to make repetitive connections between the studied concepts.

When considering difficult notions Ref.[3] states that: "one form of presentation is not enough, even for a small set of students". The assessment of such style of teaching, undertaken during several years by the authors of Ref.[4], has shown improvement of students' grades by $16-20$ $\%$.

\section{Goals and learning outcomes}

The major goal of this project is to develop a conceptually new approach in the teaching of a one-semester EFW course for electrical engineering juniors and establish a new EFW undergraduate lab. Through lab experiments, educational Java applets, and software for numerical solution the undergraduate students will actively acquire understanding of the theoretical principles of EFW and develop a scientific approach, which promotes critical 
reasoning and creative thinking. To achieve these specific goals, the following approach will be pursued:

(a) A new undergraduate lab for teaching EFW with seven stand-alone stations (each of them fully equipped to carry out experiments) will be established;

(b) A set of seven experiments on EFW will be introduced;

(c) A set of power-point lectures, with emphasis on EFW as an experimental science, will be developed;

(d) A new textbook, lab manual, and other teaching materials will be developed, published, and widely disseminated;

(e) Day-to-day monitoring of students' learning progress, by using the Blackboard online system, will be established;

(f) The effect of the novel teaching style on students' learning of EFW will be assessed and evaluated.

The teaching strategies employed in the new course, the creation and implementation of lab activities specifically designed to address key concepts considered in course lectures, the incorporation of Java applets, and the numerical simulations, will be demonstrable through the students' ability to:

1. Carry out a set of diverse lab experiments on EFW and to analyze the obtained data. Detailed records for the EFW lab experiments will be kept. The number of students successfully completing each lab, as well as an item analysis of the lab grades will be created. The analysis will enable the course instructors to determine both the least and the most challenging aspects of each laboratory; students will respond to (brief) surveys at the conclusion of each activity. The lab activities and/or the lectures will be adjusted in light of the data and findings. Student analysis of data will be a component of the scoring rubric; data analysis skills will be documented in the lab activities as well as in course quizzes and exams.

2. Connect what they learned in the lab with the contents of the lectures in a coherent way. Students will be asked on formal assessments to relate their learning from lab and lecture experiences through the presentation of real-world data sets and problems. (Students may be presented with data and asked to relate the data to the theoretical model; they may be asked to explain anomalies in the data that they collected.) The Blackboard system used at UB will provide a formative feedback for instructional changes; formal classroom assessments will provide summative feedback about overall student abilities.

3. Apply the theory of EFW to engineering problems. Students will be presented with novel engineering issues that they will be asked to relate to the current theoretical models that have been discussed in class. Once again, this information will be obtained through the use of both the Blackboard system and formal assessment (quizzes, exams) measures.

4. Use computer simulation programs to obtain numerical results and compare them with the analytical solutions as well as with the results obtained in lab experiments. Students will be presented with the problems (either for homework, in class, or on assessments) that ask them to relate results obtained by simulations as well as analytical and experimental analyses. Blackboard system data will be collected to demonstrate pre- and post-instructional responses, thus providing evidence of instructional impact.

5. Apply the acquired practical skills to carry out a group design project. Students will 
participate in a small group design project; individual participation will be documented throughout the course of the project. Team members will evaluate both their contribution and the contributions of their team members (see the evaluation form in the Appendix) during the group project. The evidence from the design project will be analyzed and summarized accordingly.

\section{Topics of lecture component}

The understanding of modern electricity and magnetism requires a certain level of mathematical knowledge. In the new course the concepts of vector algebra and vector calculus will be introduced and discussed gradually in correspondence with teaching material covered in the course. Our and other instructors' teaching experiences $[5,6]$ show that the students are more likely to learn abstract mathematical notions when these notions are applied to specific experimental problems.

The fourteen-week one-semester lecture/lab course with seven main topics is currently being developed. Each topic is covered during two weeks and is accompanied by a corresponding lab experiment. For better learning of new abstract notions corresponding Java applets will be used from several websites such as: http://www.amanogawa.com/ and http://www.falstad.com/ mathphysics.html. The lecture part of the course will begin with a short two-week review of the properties electromagnetic fields and waves in vacuum that the students learned from the prerequisite courses PHY 108 and PHY 207. In the proposed course, the divergence and curl of a vector field and proofs of Gauss and Stokes theorems with emphasis on the physical meaning of the divergence and curl will be introduced first. After this, the four Maxwell's equations in differential form will be presented and simple electrostatics problems will be discussed. We will consider the two equations of electrostatics: the equation for curl $\mathbf{E}$ represents the potential character of an electrostatic field and allows introduction of a scalar electric potential, while the equation for $\operatorname{div} \mathbf{E}$ gives the source of the electric field $\mathbf{E}$. In magnetostatics we will consider also two equations: the equation for $\mathbf{B}$ has zero divergence indicating that there is no magnetic analogue for the electric charge; whereas the curl of $\mathbf{B}$ is nonzero indicating that magnetic field occurs only in the presence of an electric current. Having this very compact presentation of electrostatics and magnetostatics we will be able to include some of the modern electromagnetic topics such as "Fiber optic waveguides" and "Waves in layered structures and superlattices". The central part of the proposed course (Parts II - IV) covers time-varying fields with emphasis on plane-wave propagation. We will consider interference and space and time coherence. Energy, momentum, and angular momentum associated with electromagnetic waves will be considered in detail. Using a unified description of electromagnetic waves we can discuss other topics such as: electromagnetic waves in optically active media, electromagnetic waves in guided and periodic structures, non-linear optics, and fiber optics.

\section{Set of seven EFW experiments}

The teaching of EFW to undergraduate students is a very challenging task $[7,8]$ and a new approach based on lab experiments is needed. The planned seven lab experiments will serve as a basis for the lab component of the novel teaching style and for students' active learning experience. It should be noted that students' active learning also will be provided by Java applets which help the students to visualize the electrical and magnetic phenomena. Surveys on educational Java applets [3,9] show that they are very good aids for active learning as they 
improve comprehension and engagement. Numerical simulation is also an important tool of contemporary science and engineering and for this reason special courses on computer simulation have been introduced in many engineering colleges. The incorporation of computer simulation, Java applets, and lab experiments into the teaching of the new EFW course will help students acquire abstract electricity and magnetism concepts on a higher level of comprehension.

Here we shortly describe the experiments that constitute the lab component of the proposed course. In Labs 1, 2, and 5 students will use equipment from PASCO Scientific (seven sets of equipment cost \$12,500). In Labs 4, 6, and 7 the equipment from Klinger Educational Products will be used (seven sets of equipment cost $\$ 46,500$ ). The cheapest and the easiest to organize is Lab. 3 which introduces students waves in optically active media (seven WXG-4 disc polarimeters cost $\$ 2,200)$. To give an idea how the lab component of the course will be organized we give more detailed description of Lab 3.

Lab. 1 "Electromagnetic waves". The students will explore several properties of electromagnetic waves. They will use a $15 \mathrm{~mW}$ microwave transmitter (frequency $f=10.525$

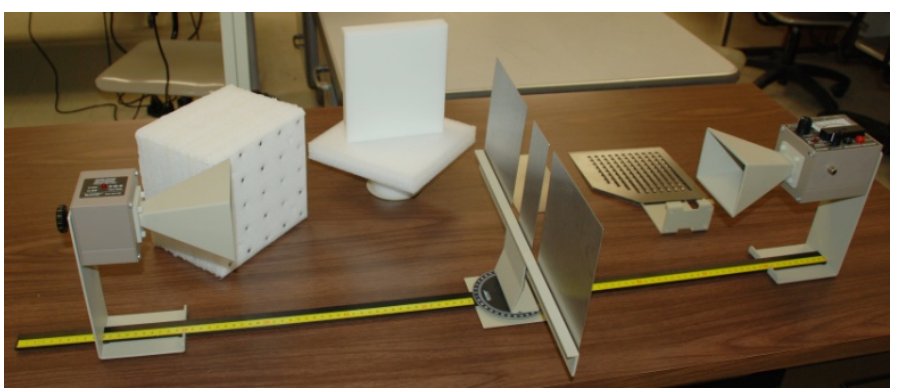

Fig. 1. The experimental setup for carrying out experiments in Labs 1, 2, and 5. $\mathrm{GHz}$, wavelength $\lambda=2.85 \mathrm{~cm}$ ) equipped with a resonant cavity and a horn antenna. The microwave beam intensity will be measured using a receiver that consists of a Schottky diode in a 10.525 GHz resonant cavity and an amplifier.

(a) Polarization properties of electromagnetic waves. The students verify that the emitted radio waves are linearly polarized along the axis of the transmitter diode and that the receiver acts as an analyzer with the polarization

axis parallel to the axis of the receiver diode (Fig. 1). They will place the detector diode at an angle $\varphi$ to the transmitter diode and measure the intensity $I$ of the received signal as a function of $\varphi$. Then, they will analyze the data and verify Malus's law: $I=I_{0} \cos ^{2} \varphi$. Finally, the students will verify that a wire grid can act as a polarizer or an analyzer for microwaves.

(b) Standing waves. The setup of Fig.2 will be used. The transmitter sends a wave along the rail on which the various components are

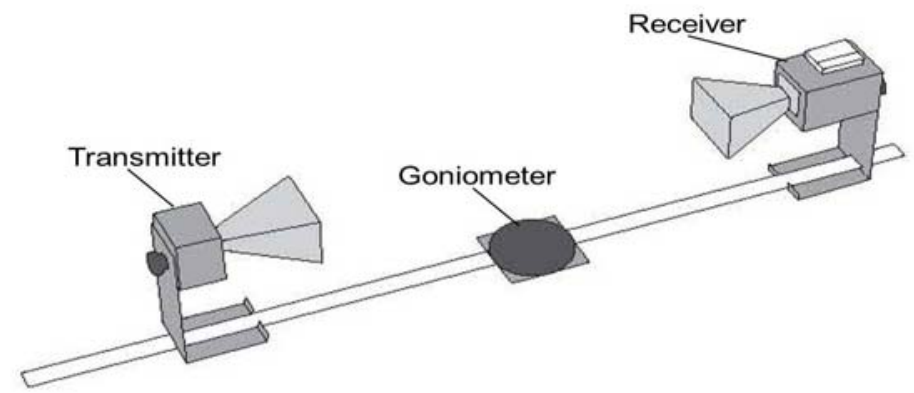

Fig. 2. The experimental setup for studying standing waves. mounted. At the other end of the rail a reflector is placed with its plane perpendicular to the emitted wave. The emitted and reflected waves form a standing wave pattern with a series of alternating intensity minima (nodes) and maxima (antinodes). The intensity of the standing wave is measured using a detector probe that can be positioned anywhere between the transmitter and the reflector. The distance between two consecutive nodes or two consecutive antinodes is equal to $\lambda / 2$, while the distance between a node and the next antinode is equal to $\lambda / 4$. 
The students will measure the wavelength and carry out an error analysis.

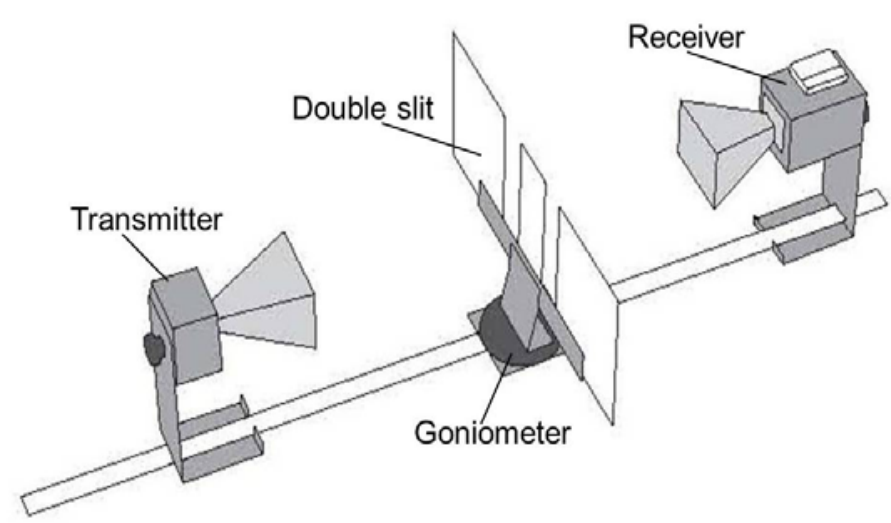

Fig. 3. Double-slit experimental setup. (c) Double-slit interference experiment. The electromagnetic wave emitter will generate a wave perpendicular to a double slit with a slit center separation of $8 \mathrm{~cm}$ (Fig. 3). The receiver will be placed behind the double slit, on the rotating arm of a goniometer so that the angle $\theta$ between the incident beam and the beam transmitted through the double slit can be directly measured. The transmitted beam consists of the superposition of the two beams that pass through each slit which interfere to generate a series of alternating maxima (constructive interference) and minima (destructive interference). The transmission angles of the intensity maxima are given by the equation: $d \sin \theta=m \lambda$, where $m= \pm 1, \pm 2, \pm 3, \ldots$ is the order of diffraction. In this experiment the students will observe two intensity maxima at $\theta= \pm 21^{\circ}(\mathrm{m}=$ $\pm 1)$ and a $\theta= \pm 45^{\circ}(m= \pm 2)$. An error analysis comparison between the $m= \pm 1$ and $m= \pm 2$ will be carried out.

Lab. 2 "Electromagnetic waves at the interface between two media". The students will

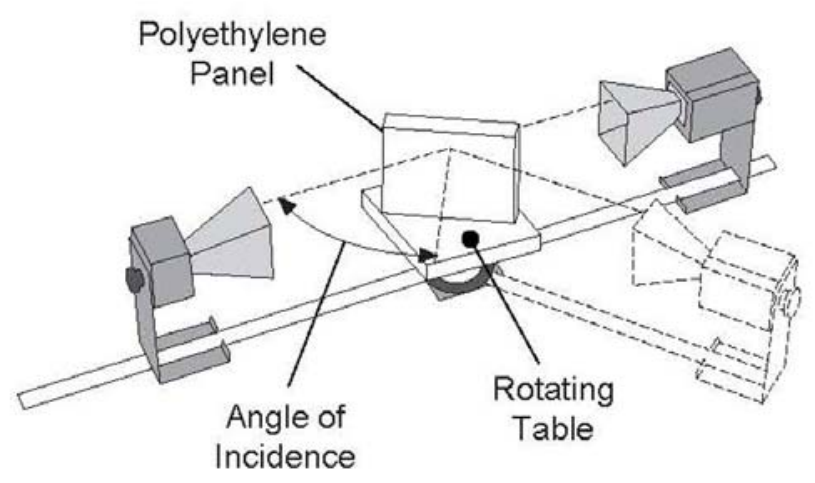

Fig. 4. Experimental setup for finding Brewster's angle $\theta_{\mathrm{B}}$. determine Brewster's angle for a microwave beam reflected by a polyethylene slab (Figs. 1 and 4). The intensity of the microwave beam will be measured as function of angle of incidence $\theta$ when the polarization of the electromagnetic wave lies in the plane of incidence (horizontal polarization). The intensity will vanish when the reflected beam (reflection angle $=\theta_{\mathrm{B}}$ ) direction is perpendicular to the direction of the transmitted beam. In this case the induced electric dipole moment of the polyethylene molecules is parallel to the direction of the reflected wave and for this reason there is

no reflected beam. The angle $\theta_{\mathrm{B}}$ is known as "Brewster's angle" and its value is given by the equation: $\tan \theta_{\mathrm{B}}=n$, where $n$ is the refractive index of the polyethylene slab.

\section{Lab. 3 "Electromagnetic waves in optically active media".}

I. Objectives: To explore the interaction of chiral molecules with linearly polarized light. The students will prepare sugar solutions of different concentration. They will use a polarimeter illuminated by a sodium lamp (wavelength $\lambda=590 \mathrm{~nm}$ ) to: (a) verify that the sugar solution rotates the polarization plane of the transmitted light in the clockwise direction (dextrorotatory) and turpentine rotates the polarization plane of the transmitted light in the counter-clockwise direction (levorotatory); (b) measure the rotation angle $\theta$ of the polarization plane of the incident 
linearly polarized light beam that is transmitted through a length $l$ of the sugar solution and turpentine.

II. Equipment: WGX-4 disc polarimeter

Specifications:

Measurement range: $\pm 180^{\circ}$

Accuracy: $\quad \pm 0.05^{\circ}$

Weight: $\quad 7.6 \mathrm{~kg}$

Dimensions: $51 \mathrm{~cm} \times 13.5 \mathrm{~cm} \times 25 \mathrm{~cm}$

\section{Introduction}

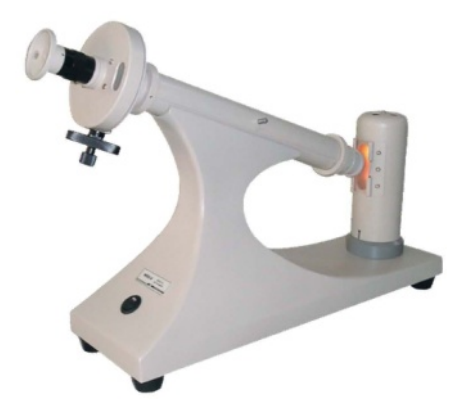

WXG-4 disc polarimeter

A variety of crystalline solids, liquids, and solutions of crystalline substances, known as

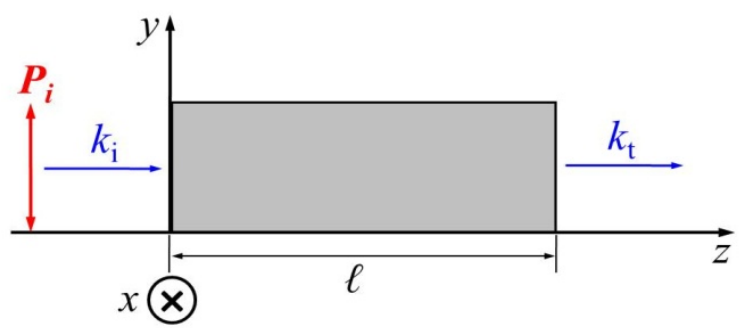

Fig. 5. Illustration of linearly polarized light incident on medium of length $l$.

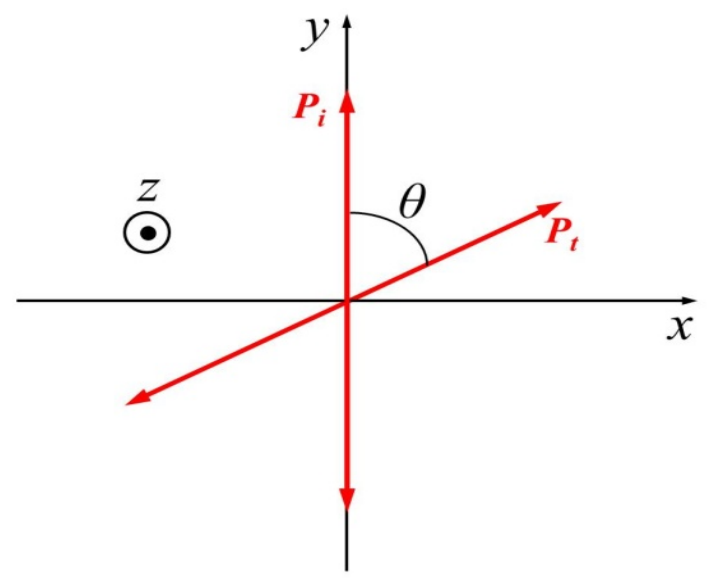

Fig. 6. Rotation of polarization plane of incident light by an angle $\theta$ after light transmits optically active medium. "optically active", exhibits the ability to rotate the polarization plane of incident light which is linearly polarized. This is illustrated in Fig. 5. Monochromatic light propagates along the $z$-axis and is transmitted through a column of length $l$ of an optically active substance. The incident light is linearly polarized along the $y$-axis. The transmitted light is also linearly polarized but its polarization plane has been rotated by an angle $\theta$. This is shown in Fig. 6. The red double-headed vertical arrow along the $y$-axis marked as $\boldsymbol{P}_{\boldsymbol{i}}$ represents the polarization direction of the incident light. The tilted red arrow marked as $\boldsymbol{P}_{\boldsymbol{t}}$ shows the polarization of the transmitted light beam. We note that the polarization plane is defined by the polarization direction and the propagation direction. In Fig. 6 we are looking in the direction of the incoming light (the $z$-axis) and the polarization plane of the transmitted light has been rotated in the clockwise direction by an angle $\theta$. This optically active material is called dextrorotatory or more simply $d$-rotatory. The angle $\theta$ is taken to be positive. If the rotation is counter-clockwise the optically active material is called levorotatory (or more simply $l$-rotatory). The angle $\theta$ is indicated as negative. This effect was discovered in 1811 by Dominique Arago. In 1825 Augustin Fresnel interpreted the phenomenon of optical activity by pointing out that linearly polarized light can be decomposed into two circularly polarized components (left and right circularly polarized) propagating in the same direction. In addition he assumed that the left- and right-circularly 
polarized components propagate with different velocities in the optically active medium and thus have indices of refraction equal to $n_{L}$ and $n_{R}$, respectively. He calculated the rotation angle to be:

$$
\theta=\frac{\pi l}{\lambda_{0}}\left(n_{L}-n_{R}\right)
$$

Here $l$ is the light path length inside the optically active material, and $\lambda_{0}$ is the wavelength of the incident light in vacuum. We note that if $n_{L}>n_{R}$, then $\theta>0$ and the material is $d$-rotatory. If on the other hand $n_{L}<n_{R}$, then $\theta<0$ and the material is $l$-rotatory. In the case of solutions the angle $\theta$ is given by the equation:

$$
\theta=\frac{l d}{10}[\rho]
$$

Here $l$ is the path length in centimeters, $d$ is the number of grams of the dissolved optically active substance per cubic centimeter of water, and $[\rho]$ is a constant called "specific rotation" or "rotatory power" whose value and algebraic sign is characteristic of the dissolved optically active substance. The factor of $10 \mathrm{in} \mathrm{Eq.} \mathrm{(2)} \mathrm{is} \mathrm{to} \mathrm{compensate} \mathrm{for} \mathrm{the} \mathrm{fact} \mathrm{that} \mathrm{the} \mathrm{liquid} \mathrm{cell}$ length is $1 \mathrm{dm}=10 \mathrm{~cm}$.

\section{Experimental Method}

As schematic diagram of the equipment used in this experiment is shown in Fig. 7. A light source produces a collimated

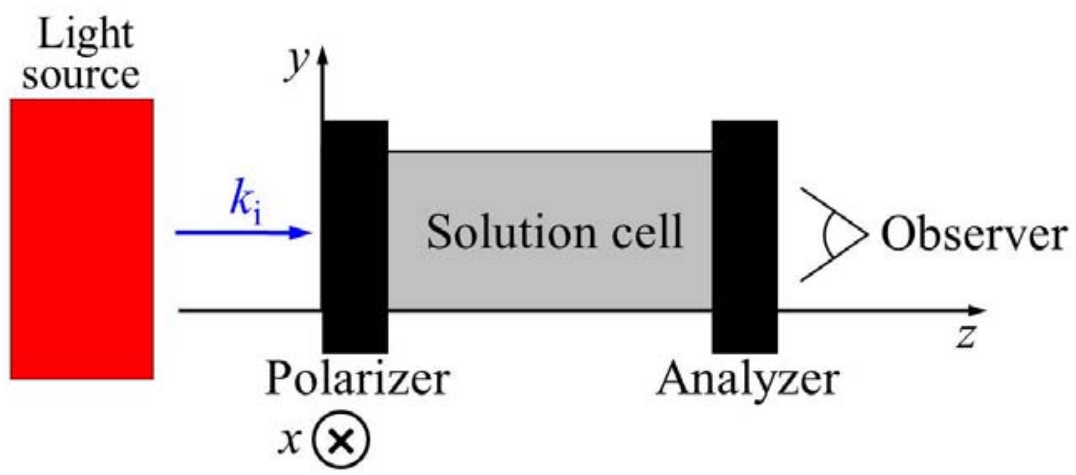

Fig. 7. A schematic diagram of the experimental setup for measurements of rotation angle $\theta$. light beam that is incident at the left side. The incident beam passes through a polarizer and becomes linearly polarized along the $y$ axis. The light propagates along the solution cell axis and is analyzed by the analyzer at the right of the cell. The observer detects the intensity of the transmitted light.

The instrument is operated as follows. The analyzer is mounted on a rotational stage so that the angle $\varphi$ between the polarization axis of the analyzer and the axis of the polarizer can be set at any value.

1. An empty solution cell is placed in the polarimeter. The angle $\varphi$ is set at $90^{\circ}$ by rotating the analyzer so that the intensity of the transmitted light is a minimum. This follows from the Malus's law:

$$
I_{t}=I_{i} \cos ^{2} \varphi
$$

2. The solution cell is now placed in the polarimeter. The intensity of the transmitted light is not at a minimum any more. This is because the solution of the optically active material has rotated the polarization plane of the transmitted light so that it is not along the $y$-axis anymore. We rotate the analyzer to a new angle $\varphi^{\prime}$ so that the intensity of the transmitted light is once more a 
minimum. If the solution is $d$-rotatory ( $l$-rotatory) we must rotate the analyzer in the clockwise (counter- clockwise) direction.

3. The rotation angle $\theta$ is given by the equation:

$$
|\theta|=\left|\varphi-\varphi^{\prime}\right|
$$

Its algebraic sign is positive (negative) if the analyzer was rotated in the clockwise (counterclockwise) direction.

\section{Procedure}

V1. Use the solution cell filled with turpentine. Measure the rotation angle $\theta_{1}$.

V2. Use the solution cell with sugar solution of known concentration. Measure the rotation angle $\theta_{2}$.

V3. Use the solution cell with sugar solution of unknown concentration. Measure the rotation angle $\theta_{3}$.

\section{For the report}

VI1. Compare $\theta_{1}$ measured in section $\mathbf{V 1}$ with the accepted value of $-37^{\circ}$.

VI2. From the value of $\theta_{2}$ measured in section V2 determine [ $\rho$ ] for sugar solution using Eq. (2). Compare your experimental value with the accepted value of $66.4 \mathrm{deg} / \mathrm{dm}$.

VI3. Using Eq. (2) and the value of $\theta_{3}$ measured in section $\mathbf{V 3}$ determine the concentration $d$ of the unknown sugar solution. Ask the TA what the value of $d$ was. Compare this with your experimental result.

Lab. 4 "Propagation of electromagnetic waves on a transmission line". The students will use a UHF emitter $(f=434 \mathrm{MHz})$ to propagate an electromagnetic wave down a transmission line (Fig. 8). Initially the transmission line will be shorted so that the electromagnetic wave will be reflected at the end of the line and interfere with the incident wave thus forming a standing wave. The students will use two types of detectors to find the maxima of the electric and magnetic

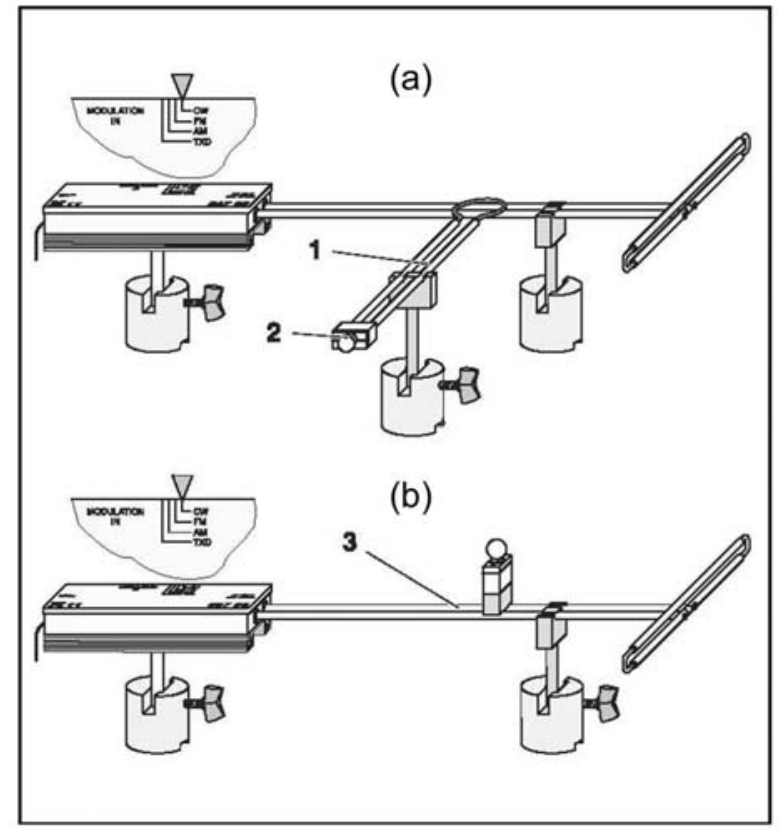

Fig. 8. Experimental setup for finding: (a) magnetic field maxima and (b) electric field maxima on a transmission line. fields $\boldsymbol{E}$ and $\boldsymbol{B}$ along the transmission line. One detector is connected across the transmission line and has a light bulb which detects the maxima in voltage (and thus of the electric field).The second detector is coupled inductively to the transmission line and detects the maxima of the current (and thus of the magnetic field). The adjacent maxima of the $\boldsymbol{E}$-field and $\boldsymbol{B}$-field are separated by a distance equal to $\lambda / 2$. The separation of adjacent $\boldsymbol{E}$-field and $\boldsymbol{B}$-field maxima is equal to $\lambda / 4$, i.e. when the $\boldsymbol{E}$-field has a maximum the $\boldsymbol{B}$-field has a minimum and vice versa. The standing wave pattern is not formed if the transmission line is terminated with a $200 \Omega$ resistor. In this case there is no reflected wave at the end of the transmission line. A $\lambda / 2$ dipole emission antenna will then be placed at the end of the transmission line and the $\boldsymbol{E}$-field and $\boldsymbol{B}$-field 
maxima will be detected. The antenna has two $\boldsymbol{E}$-field maxima at the ends of the antenna while the $\boldsymbol{B}$-field has a single maximum at the antenna center. The standing wave pattern on the transmission line is not present as long as the dipole antenna is connected.

Lab. 5 "Diffraction of electromagnetic waves by a periodic structure". The students will explore the Bragg diffraction of a monochromatic microwave beam (frequency $f=10.525 \mathrm{GHz}$, wavelength $\lambda=2.85 \mathrm{~cm}$ ) by a three-dimensional cubic lattice formed by $1 \mathrm{~cm}$ diameter metallic spheres embedded in a dielectric matrix (see Fig. 1). The separation $d$ of the spheres is equal to $3.8 \mathrm{~cm}$. This is a macroscopic analog of a crystal in which the metal spheres scatter the incident beam just as atoms scatter X-rays in a crystal. The experiment is carried out on a rotating platform, on which the cubic lattice is placed, equipped with a rotating arm on which the receiver is located. The students will study Bragg's law: $2 d \sin \theta=m \lambda$. Here $\theta$ is the angle between the incident and the diffracted beam and the face of the scatterer; $m$ is the order of diffraction. Two Bragg peaks are expected at $\theta=22^{\circ}(m=1)$ and $\theta=48^{\circ}(m=2)$.

Lab. 6 “Radiation pattern of electromagnetic waves formed by a dipole antenna". An ultrahigh frequency emitter $(f=434 \mathrm{MHz})$ will be used to drive an emitter dipole antenna of length

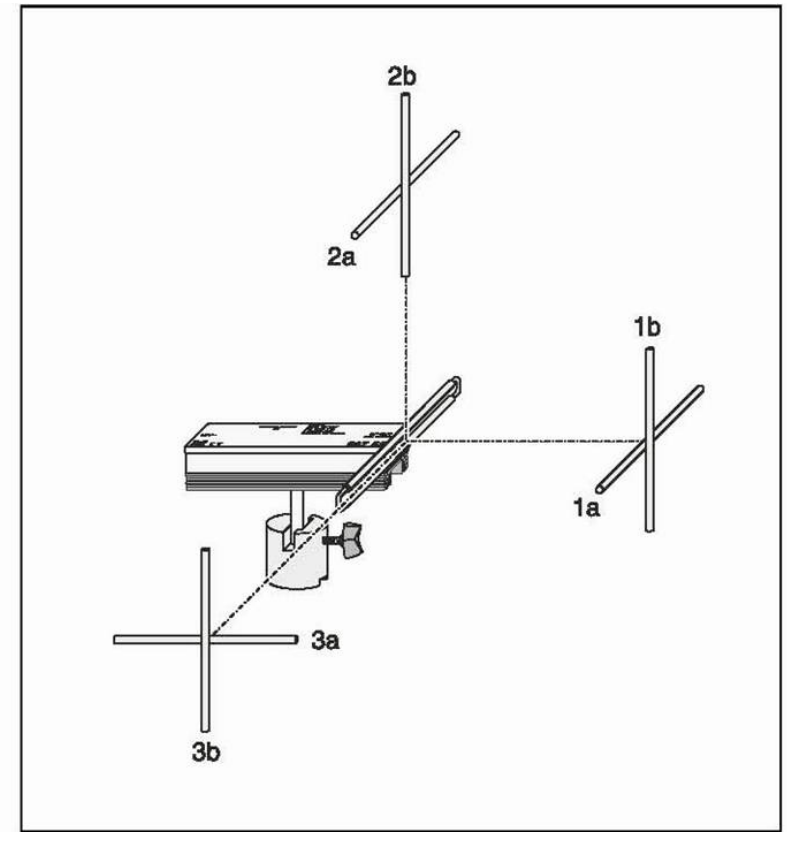

Fig. 9. Experimental setup for finding radiation pattern of electromagnetic waves emitted by a dipole antenna. $\lambda / 2$. The emitted electromagnetic wave will be detected by the second receiver antenna of the same length and associated electronics. The students will explore the emission characteristics of the dipole antenna. More specifically they will verify that: (a) Maximum emission occurs along a direction perpendicular to the dipole antenna axis; (b) The emitted electromagnetic wave is linearly polarized perpendicular to the propagation direction. This will be accomplished by rotating the receiver antenna. The students will find the orientations for maximum signal (receiver antenna axis is parallel to polarization) and minimum signal (receiver antenna axis is perpendicular to polarization); (c) No electromagnetic waves are emitted along the emitter antenna axis (Fig. 9). Finally the students will map the intensity of the receiver signal as a function of the angle between the dipole antenna axis and the electromagnetic wave propagation direction.

Lab. 7 "Modulation and demodulation of electromagnetic waves". The students will study the amplitude modulation and demodulation of electromagnetic waves $(f=434 \mathrm{MHz})$ by an audio signal in the $100 \mathrm{~Hz}-10 \mathrm{kHz}$ frequency range. This is a dramatic experiment in which the students will transmit a modulated radio signal, receive it a few meters away, demodulate it, and listen to the audio signal from a loud-speaker. It will be emphasized that this amazing observation is possible only due to the displacement current term added by Clark Maxwell in the Ampere's law. "The caterpillar has turned into a butterfly!" is how Richard Feynman beautifully described the process of radiation of electromagnetic waves from a source in his now classic "Lectures on Physics" [10]. 
During the course students organized in groups of three at the beginning of the course will carry out one small design project on electromagnetic fields and waves. Several groups can work on the same type of project and compare the results at the end of the course. The students will write a report on their results at the end of the project and give a presentation in the form of a talk during which they will demonstrate the devices which they have built. Examples of some possible projects that students may choose are: (a) Design and build simple radio transmitter; (b) Design and build a grating spectrometer; (c) Design and construct Yagi or other antenna types;

(d) Design and build spatial TE/TM polarization splitters for integrated optical components; and

(e) Design and build optical dielectric waveguide.

\section{Formative evaluation tools}

Conceptual tests are under development to assess student learning of electromagnetic fields and waves. The pre-test will include approximately 15 items to assess students' general understanding of electromagnetic fields and waves; the post-test will include the original 15 items as well as 30 additional items that will more fully assess gains in students' understanding. Students completing a traditional course will serve as a control population for the test measures. The pre-test will be given in the beginning of the semester and the post-test will be given at the end of the semester. Additionally, two surveys - "Survey of Student Interest toward Electromagnetic Fields and Waves (SSEMFW)," and "Evaluation of Effectiveness of Electromagnetic Fields and Waves Course Materials (EEEFW)" will be given to students and to course instructors, respectively.

Formative evaluations are under development for each of the laboratory experiments and Java applets. These evaluations will be given to students following the completion of the specific laboratory experiments and Java virtual labs. The assessments will measure student perceptions of the presentation of the laboratory content, the content validity and the level of student engagement. The feedback will be used to refine the experiments and applets.

Six weeks before the end of the semester, students will be given comprehensive problems (design projects) to assess their ability to apply electromagnetic fields and waves principles in an authentic context. The complete array of assessments will assess students' theoretical and practical understanding of electromagnetic fields and waves.

\section{Conclusion}

A new EFW one-semester course for undergraduate engineering students is being developed which is characterized by: 1) a nontraditional synergistic style of teaching 2) the introduction of important notions of electricity and magnetism on the basis of corresponding experiments; and 3) a unified description of the entire spectrum of electromagnetic waves to address interdisciplinary issues. The important aspect of this new course is the radical change of the teaching style of the traditional course on EFW. Its main features are: (a) the hands-on experience provided to students while carrying out seven lab experiments, (b) day-to-day monitoring of students' learning process by using Blackboard online system, and (c) assessment of progress in learning through low-stake quizzes, homework problems, lab reports, and exams. 


\section{Acknowledgement}

This research was supported by NSF Grant No. DUE-1140718.

\section{Bibliography}

1. N. Nirmalakhandan, C. Ricketts, J. McShannon, and S. Barrett, "Teaching tools to promote active learning: case study", J. Prof. Issues Eng. Educ. and Pract. 133, No. 1, pp. 31 - 37 (2007).

2. M. P. Clough and K. J. Kauffman, "Improving engineering education: A research-based framework for teaching”, J. Eng. Educ. 88, No. 4, pp. 527 - 534 (1999).

3. G. Crisp, "Interactive online learning and assessment", EDUCAUSE IN AUSTRALASIA 2003, 6-9 May 2003, Adelaide, Australia, Conference Proceedings, pp. 840 - 848.

4. J. Masters, T. M. Madhyastha, and A. Shakouri, "Educational applets for active learning in properties of electronic materials", IEEE Trans. Educ. 48, No. 1, pp. 29 - 36 (2005).

5. "Engineering Electromagnetics", W. H. Hayt, Jr. and J. A. Buck, McGraw-Hill, New York, $6^{\text {th }}$ edition, 2001.

6. L. D. Feisel and A. J. Rosa, "The role of the laboratory in undergraduate engineering education", J. Engineering Education, 94, No 1, pp. 121 - 130 (2005).

7. D. P. Maloney, T. L. O'Kuma, C. J. Hieggelke, and A. Van Heuvelen, "Surveying students' conceptual knowledge of electricity and magnetism”, Phys. Educ. Res., Am. J. Phys. Suppl. 69, No 7, pp. S12 - S23 (2001).

8. L. Ding, R. Chabay, B. Sherwood, and R. Beichner, "Evaluating an electricity and magnetism assessment tool: Brief electricity and magnetism assessment", Phys. Rev. ST Phys. Educ. Res. 2, No 7, pp. 010105-1-7 (2006).

9. T. Naps, G. Roessling, V. Almstrum, W. Dann, R. Fleischer, C. Hundhausen, A. Korhonen, L. Malmi, M. McNally, S. Rodger, and J. A. Velázquez-Iturbide, " Exploring the role of visualization and engagement in computer science education”, SIGCSE Bull. 35, No. 2, pp. 131 - 152 (2002).

10. "The Feynman Lectures on Physics", R. P. Feynman, R. B. Leighton, and M. Sands, Addison-Wesley, 1977, Vol. 2, p. 18-8. 


\section{Appendix}

\section{Evaluation of Students' Design Project}

Directions: Rate each of the following five aspects of each design project, by darkening in the circle for Satisfactory (2), Partially Satisfactory (1), or Unsatisfactory (0). Add the total number of points. You may make notes over the gray

\begin{tabular}{|c|c|c|c|c|c|c|}
\hline Aspects & & Satisfactory & & $\begin{array}{c}\text { Partially } \\
\text { Satisfactory }\end{array}$ & & Unsatisfactory \\
\hline $\begin{array}{c}\text { 1. The solutions of the } \\
\text { problem are } \\
\text { reasonable }\end{array}$ & 2 & $\begin{array}{c}\text { The solutions are } \\
\text { both valid and } \\
\text { complete }\end{array}$ & 1 & $\begin{array}{c}\text { The solutions are } \\
\text { valid but } \\
\text { partially } \\
\text { incomplete }\end{array}$ & 0 & $\begin{array}{l}\text { The solutions are } \\
\text { invalid and/or } \\
\text { incomplete }\end{array}$ \\
\hline $\begin{array}{l}\text { 2. Correctly chosen } \\
\text { theoretical } \\
\text { justification of the } \\
\text { device operation }\end{array}$ & 2 & $\begin{array}{l}\text { The chosen } \\
\text { justification is } \\
\text { reasonable and } \\
\text { fully established }\end{array}$ & 1 & $\begin{array}{l}\text { The chosen } \\
\text { justification is } \\
\text { partially flawed } \\
\text { or not fully } \\
\text { established }\end{array}$ & 0 & $\begin{array}{l}\text { The chosen } \\
\text { justification is } \\
\text { invalid or not } \\
\text { established }\end{array}$ \\
\hline $\begin{array}{l}\text { 3. Correctly chosen } \\
\text { materials for the } \\
\text { device } \\
\text { implementation }\end{array}$ & 2 & $\begin{array}{c}\text { All of the } \\
\text { necessary } \\
\text { materials have } \\
\text { been specifically } \\
\text { identified }\end{array}$ & 1 & $\begin{array}{l}\text { Most of the } \\
\text { necessary } \\
\text { materials } \\
\text { specifically } \\
\text { identified }\end{array}$ & 0 & $\begin{array}{c}\text { Necessary } \\
\text { materials are } \\
\text { missing or have } \\
\text { not been } \\
\text { specifically } \\
\text { identified }\end{array}$ \\
\hline $\begin{array}{l}\text { 4. The procedures } \\
\text { chosen for } \\
\text { experimental setup } \\
\text { are appropriate }\end{array}$ & 2 & $\begin{array}{l}\text { The experimental } \\
\text { procedures are } \\
\text { appropriate and } \\
\text { complete }\end{array}$ & 1 & $\begin{array}{c}\text { The } \\
\text { experimental } \\
\text { procedures are } \\
\text { largely } \\
\text { appropriate or } \\
\text { largely complete }\end{array}$ & 0 & $\begin{array}{c}\text { The } \\
\text { experimental } \\
\text { procedures are } \\
\text { largely } \\
\text { inappropriate or } \\
\text { largely } \\
\text { incomplete }\end{array}$ \\
\hline $\begin{array}{l}\text { 5. Documentation is } \\
\text { complete with } \\
\text { details }\end{array}$ & 2 & $\begin{array}{l}\text { Detailed } \\
\text { documentation is } \\
\text { provided }\end{array}$ & 1 & $\begin{array}{l}\text { Detailed } \\
\text { documentation is } \\
\text { provided with } \\
\text { only minor } \\
\text { omissions }\end{array}$ & 0 & $\begin{array}{c}\text { Detailed } \\
\text { documentation is } \\
\text { lacking }\end{array}$ \\
\hline
\end{tabular}

\section{Total:}

